\title{
STRUCTURE PREDICTION AND IN SILICO DESIGNING OF DRUGS AGAINST KALLIKREIN PROTEIN 12
}

\author{
MANISH DEVGAN* \\ *Faculty of Pharmacy, R. P. Educational Trust Group of Institutions, Bastara, Karnal 132001, Haryana, India \\ Email: manishdevgan12@gmail.com
}

Received: 27 Nov 2016, Revised and Accepted: 23 Jan 2017

\begin{abstract}
Objective: Human Kallikrein protein 12 (hK12) might serve as a novel diagnostic and prognostic biomarker, as well as a potential therapeutic target, in gastric cancer.

Methods: In this work, a theoretical model of hK12 receptor protein was generated using the concepts of homology modeling and loop modeling. The resulting model was validated with Ramachandran plot analysis. The ligands generated with the help of Drug bank were docked against hK12 receptor protein using AutoDock Vina in PyRx 0.8. The structure of ligand DB04786 (Suramin), with least binding energy, was varied by using ACD/ChemSketch 8.0 and the docking was done for the resulting 16 new ligands.

Results: The results indicated that the ligand10 bears the minimum binding energy ( $-12.3 \mathrm{Kcal} / \mathrm{mol})$ with the target protein and thus the prospects of binding are high. The results also clearly demonstrated that the in silico molecular docking studies of selected ligands, i.e., suramin, ligands 5, 6,
\end{abstract} 10 and 16 with hK12 protein exhibited favourable binding interactions and warranted.

Conclusion: Further studies needed for the development of potent inhibitors for the overexpression of hK12 protein making the management of gastric cancer more efficient.

Keywords: Gastric cancer, Kallikrein protein, Docking, Homology modelling, Human kallikrein 12

(C) 2016 The Authors. Published by Innovare Academic Sciences Pvt Ltd. This is an open access article under the CC BY license (http://creativecommons.org/licenses/by/4.0/] DOI: http://dx.doi.org/10.22159/ijcpr.2017v9i2.17387

\section{INTRODUCTION}

Gastric cancer (GC) is the fourth leading cancer in the world and the second most common cause of death [1]. The risk factors for gastric cancer include male gender, cigarette smoking, Helicobacter pyroli infection, atrophic gastritis and partial gastrectomy. A small number of patients may have a genetic propensity syndrome [2]. The focus is not only on the well-established targeted treatments for GC, like anti-epidermal growth factor receptor therapies, Anti-HER2 (ERBB2) therapy, angiogenesis pathways, hepatocyte growth factor/c-MET signalling pathway and programmed cell death-1 receptor and its ligands but also on the determination of predictive markers and co-development of drugs with these markers [3]. Human tissue kallikreins (hKs) are secreted serine proteases with diverse expression patterns and physiological roles. The entire human kallikrein gene locus was discovered and found to contain 15 kallikrein genes around chromosome 19q13.2-q13.4. The human kallikrein-related peptidases are the most efficient cancer biomarker ever employed. Kallikrein genes are expressed abnormally in various malignancies, where they affect cancer-cell growth and metastasis. Their deregulated expression pattern, often associated with various clinicopathological characteristics of cancer patients, can be exploited, solely or within multiparametric panels, as a prognostic biomarker [4-7]. A study concluded that positive kallikrein expression seems to be associated with worse OS and PFS in patients with ovarian cancer [8]. Yang et al. (2015) analyzed the expression and distribution of human kallikrein 5 [hK5] in triplenegative breast cancer [TNBC] tissues and came to the conclusion that the expression level of hK5 in tumour stromal cells is a promising biomarker for poor prognosis in TNBC [9]. The human kallikrein 6 gene is markedly over-expressed in gastric cancer tissue and its expression status may be a powerful prognostic indicator for patients with gastric cancer [10]. Another study suggested that kallikrein 10 expression is up-regulated in colorectal cancer and GC and higher expression of kallikrein 10 closely correlates with advanced disease stage, which predicts a poorer prognosis [11]. Wen et al. (2011) indicated in a study that kallikrein 11 expressions was reduced in gastric cancer and might serve as a novel independent prognostic marker [12]. One study indicated the possible clinical utility of kallikrein 13 as a new tumor biomarker capable of anticipating a favorable outcome for gastric cancer patients [13]. Memari et al. (2007) observed the higher levels of hK12 in malignant prostatic glands indicating a potential role during prostate carcinogenesis [14]. Another study indicated that the expression of hK12 is down-regulated at the mRNA level in breast cancer tissues and is up-regulated by steroid hormones in breast and prostate cancer cell lines [15]. Zhao et al. (2012) in a study observed that hK12 was remarkably over-expressed in GC tissues and that high hK12 expression levels were associated with the lymph node metastasis, histological type, pathological stage and poor patient prognosis. The study also exhibited that knockdown of hK12 expression leads to diminished proliferation and migratory ability with little effect on invasiveness in MKN-45 GC cells. Consequently, hK12 might serve as a novel diagnostic and prognostic biomarker, as well as a potential therapeutic target, in GC [16].

Computer Aided Drug Designing is fast becoming an important tool in Drug discovery, the in silico study has provided a new understanding of the interaction between receptor and ligands. The structure-based drug design (SBDD) methods, such as molecular docking and de novo drug design, depend on the knowledge of the structure of the target macromolecule, which are mainly obtained from crystal structures, NMR data and homology models [17].

Homology modeling estimates the 3-D structure of a given protein sequence (target) based principally on its alignment to one or more proteins of known structures (templates). The homology modeling has been widely used to predict the protein structure [18-21].

In this study, the structure of human kallikrein 12 protein was designed by using homology modeling. The docking of the ligands was done to predict the binding orientation of small drug molecules with their protein target (hK12) in order to predict the affinity and activity of the small molecules in inhibiting hK12 so that it may lead to diminished proliferation and migratory ability of gastric cancer cells. 


\section{MATERIALS AND METHODS}

The hardware used for calculating molecular modeling includes a personal computer with Intel (R) Core (TM) i3 CPU processor, Windows 7 Home Premium 32-bit operating system having RAM of $2.00 \mathrm{~GB}$.

\section{Sequence alignment}

\section{Fast alignment (FASTA)}

The FASTA format is a text-based format for representing either nucleotide sequences or peptide sequences, in which nucleotides or amino acids are represented using single letter codes. A sequence in FASTA format begins with a single-line description, followed by lines of sequence data. The description line is distinguished from the sequence data by a greater-than (">") symbol in the first column [22]. The FASTA sequence of hK12 was acquired from the website of National Centre for Biotechnology Information [23].

\section{Basic local alignment search tool (BLAST)}

The BLAST is an algorithm for comparing primary biological sequence information, such as the amino acid sequence of different proteins or the nucleotides of DNA sequences [24]. Using the FASTA sequence, the standard protein BLAST was realized on the NCBI. The protein data bank proteins database was selected and the BLAST-P was executed [25].

\section{Three-dimensional position-specific scoring matrix (3D-PSSM)}

The 3D-PSSM is a fast web-based method for protein fold recognition using $1 \mathrm{D}$ and $3 \mathrm{D}$ sequence profiles coupled with secondary structure and solvation potential information. The FASTA sequence was submitted to 3D-PSSM for fold recognition [26].

\section{Protein homology/analogy recognition Engine (Phyre)}

Phyre is a web-based service for protein structure prediction. Phyre is widely used by the biological community, with $>150$ submissions per day, and provides a simple interface to results. Phyre takes 30 min to predict the structure of a 250-residue protein [27]. The FASTA sequence was submitted to Phyre for amino acid sequence prediction [28].

\section{Templates preparation}

The data obtained from combined BLAST, 3D-PSSM and Phyre was committed to RCSB protein data bank. The Protein Data Bank (PDB) archive is the single worldwide repository of information about the 3D structures of large biological molecules, including proteins and nucleic acids [29]. The templates were selected on the basis of their resolution $(\AA)$ and R-value. All the above templates were introduced by X-ray crystallography method in PDB.

\section{Molecular modeling}

Homology modeling of hK12 was done by using EasyModeller. EasyModeller is a graphical user interface to Modeller program. It is a standalone tool in windows platform with Modeller and Python preinstalled $[30,31]$. The Swiss-Pdb viewer, an application that provides a user-friendly interface allowing analysing several proteins at the same time, was installed [32].

\section{Structure prediction}

The six templates were introduced to the EasyModeller and were aligned. The Discreet Optimized Protein Energy (DOPE) score is a statistical tool to evaluate homology models in protein structure prediction. The model with the minimum score can be chosen as the best feasible structure.

\section{Validation of predicted model}

The validation of all the five models was performed by submitting the PDB files to PDBsum. The PDBsum is a pictorial database that provides an at-a-glance overview of the contents of each 3D structure deposited in the Protein Data Bank. PDBsum provides summary information about each experimentally determined structural model in PDB. The Ramachandran plot validated the result. The residues in the most favoured region are at maximum, and those in the generously allowed and disallowed regions are at $\mathrm{a}$ minimum [33, 34].

\section{Loop modeling}

The loop regions in the given protein are normally responsible for active and binding sites. The coordinate file was submitted for loop optimisation to ModLoop, i.e., Modeling of Loops in Protein Structures, which is an automated modeling technique that significantly improves the accuracy of loop predictions in protein structures. The resulting coordinate file was directed back by e-mail. This structure was validated with the help of Ramachandran plot using PDBsum/Rampage. The process of loop modeling and successive validation was carried on until an optimized structured model of protein was obtained [35-38].

\section{Ligand generation}

The Drug bank is a unique bioinformatics/cheminformatics instrument that integrates exhaustive drug data with meticulous drug target information. The Drug Bank was used online; the FASTA sequence of the target protein was entered, and the compounds which interact with the entered FASTA were saved $[39,40]$.

\section{Molecular docking}

Virtual screening, especially the structure-based virtual screening, has emerged as a reliable, cost-effective and timesaving technique for the discovery of lead compounds [41]. Molecular docking is an important tool in structural molecular biology and computerassisted drug design. Docking is commonly applied to drug design efforts, especially high-throughput virtual screenings of small molecules, to identify new compounds that bind to a given target [42]. Before going for docking, the macromolecule and the ligands were prepared by using Pymol and ChemBio3D software $[43,44]$. The molecular docking was done against hK12 receptor protein using AutoDock Vina in PyRx $0.8[45,46]$. The grid dimensions were maximized and the parameters used were:

\begin{tabular}{ll}
\hline Centre coordinates & Dimensions $(\AA)$ \\
\hline$X=-8.6072$ & $\mathrm{X}=76.9632$ \\
$\mathrm{Y}=-0.4857$ & $\mathrm{Y}=78.1252$ \\
$\mathrm{Z}=-29.6253$ & $\mathrm{Z}=56.0648$ \\
\hline
\end{tabular}

The preeminent compound was preferred on the ground of binding energy/binding affinity (Kcal/mol) and the root mean square deviation (upper bound and lower bound).

\section{Ligand designing and docking}

The chosen ligand was utilised to draft 16 new molecules with the help of ACD/ChemSketch 8.0 freeware. The Lipinski's rule of five was used as a reference to decide the theoretical effectiveness of the drugs. These sketched structures were then exposed to energy minimization by using ChemBio3D as done before. The molecular docking of these 16 sketched molecules was done against the hK12 receptor protein by using AutoDock Vina in PyRx 0.8, the coordinates and dimensions remaining same as before.

\section{RESULTS AND DISCUSSION}

\section{Template generation}

FASTA sequence of hK12 protein was recovered from the website of NCBI. The GenBank No. is AAG23258.1 and gi no. is 10799397. It is a 254 amino acid protein. The BLAST was executed on the NCBI and 100 hits were recorded. The FASTA sequence was put through the 3D-PSSM and Phyre for prediction of protein structure. The results attained were connected and ranked in the descending order of \% ID followed by ascending order of Resolution as shown in table 1 . The six templates (1NPM, 1AOJ, 1HJ8, 2A31, 2ZPS and 4D8N) were selected on the basis of their chains, ID \%, resolution $(\leq 3 \AA)$ and the R-value $(\leq 0.5)$. 
Table 1: Generation of templates using 3D-PSSM, Phyre and RCSB protein data bank

\begin{tabular}{|c|c|c|c|c|c|c|c|c|c|}
\hline $\begin{array}{l}\text { S. } \\
\text { No }\end{array}$ & $\begin{array}{l}\text { Template/Accession } \\
\text { No }\end{array}$ & $\begin{array}{l}\text { ID } \\
\%\end{array}$ & $\begin{array}{l}\text { Resolution } \\
(\AA ీ)\end{array}$ & $\begin{array}{l}\text { R-Value } \\
\text { (Obs/Free) }\end{array}$ & $\begin{array}{l}\text { S. } \\
\text { No }\end{array}$ & $\begin{array}{l}\text { Template/Accession } \\
\text { No }\end{array}$ & $\begin{array}{l}\text { ID } \\
\%\end{array}$ & $\begin{array}{l}\text { Resolution } \\
(\AA ీ)\end{array}$ & $\begin{array}{l}\text { R-Value } \\
\text { (Obs/Free) }\end{array}$ \\
\hline 1 & 1NPM & 50 & 2.1 & 0.227 & 17 & $1 \mathrm{MBQ}$ & 47 & 1.8 & 0.195 \\
\hline 2 & $1 \mathrm{AOJ}$ & 48 & 2.5 & 0.207 & 18 & $1 \mathrm{BIT}$ & 47 & 1.83 & 0.199 \\
\hline 3 & 1HJ8 & 47 & 1.0 & 0.118 & 19 & $1 Z Z Z$ & 47 & 1.9 & 0.157 \\
\hline 4 & $1 Y 59$ & 47 & 1.2 & 0.167 & 20 & $2 Z P Q$ & 47 & 1.9 & 0.222 \\
\hline 5 & $2 \mathrm{~A} 31$ & 47 & 1.25 & 0.162 & 21 & $2 \mathrm{D} 8 \mathrm{~W}$ & 47 & 2.00 & 0.256 \\
\hline 6 & 2ZPS & 47 & 1.55 & 0.196 & 22 & 1AN1 & 47 & 2.03 & 0.228 \\
\hline 7 & $2 \mathrm{FI} 4$ & 47 & 1.58 & 0.235 & 23 & $1 B Z X$ & 47 & 2.1 & 0.238 \\
\hline 8 & 2 FI5 & 47 & 1.58 & 0.223 & 24 & 4AN7 & 47 & 2.23 & 0.246 \\
\hline 9 & $1 \mathrm{MCT}$ & 47 & 1.6 & 0.167 & 25 & 3VEQ & 47 & 2.25 & 0.274 \\
\hline 10 & $4 \mathrm{D} 8 \mathrm{~N}$ & 47 & 1.68 & 0.208 & 26 & $1 \mathrm{TAB}$ & 47 & 2.3 & 0.200 \\
\hline 11 & 1L2E & 47 & 1.75 & 0.241 & 27 & 2PSX & 47 & 2.3 & 0.269 \\
\hline 12 & 2ZPR & 47 & 1.75 & 0.223 & 28 & 3MYW & 47 & 2.5 & 0.179 \\
\hline 13 & $1 \mathrm{TGS}$ & 47 & 1.8 & 0.186 & 29 & 1TFX & 47 & 2.6 & 0.162 \\
\hline 14 & 1G3B & 47 & 1.8 & 0.193 & 30 & 2TLD & 47 & 2.6 & 0.173 \\
\hline 15 & 1TAW & 47 & 1.8 & 0.184 & 31 & $2 \mathrm{QXG}$ & 47 & 2.6 & 0.292 \\
\hline 16 & $1 \mathrm{GVL}$ & 47 & 1.8 & 0.225 & 32 & 3BSQ & 47 & 2.8 & 0.292 \\
\hline
\end{tabular}

A total of five models were generated with the help of EasyModeller and their DOPE score was obtained (table 2). Models with the lowest DOPE assessment score and Molpdf or with the highest GA341 assessment score have the most stable minimised energy. The model number 2 was selected on these bases for further analysis.

Table 2: DOPE score and Ramachandran plot statistics of the five possible models of hK12 receptor protein

\begin{tabular}{|c|c|c|c|c|c|c|c|c|}
\hline S. No. & Model No. & Molpdf & DOPE & GA341 & RMFR (No./\%) & RAAR (No./\%) & RGAR (No./\%) & RDAR (No./\%) \\
\hline 1 & B99990001 & 9602.73047 & -24414.50977 & 1.000 & $177(87.6 \%)$ & $23(11.4 \%)$ & $0(0.0 \%)$ & $2(1.0 \%)$ \\
\hline 2 & B99990002 & 8764.07324 & -25231.23438 & 1.000 & $179(88.6 \%)$ & $22(10.9 \%)$ & $0(0.0 \%)$ & $1(0.5 \%)$ \\
\hline 3 & В99990003 & 9021.87402 & -24965.33984 & 1.000 & $169(83.7 \%)$ & $27(13.4 \%)$ & $4(2.0 \%)$ & $2(1.0 \%)$ \\
\hline 4 & B99990004 & 9003.21289 & -24385.58398 & 1.000 & $176(87.1 \%)$ & $24(11.9 \%)$ & $0(0.0 \%)$ & $2(1.0 \%)$ \\
\hline 5 & B99990005 & 9768.22559 & -24535.29297 & 1.000 & $175(86.6 \%)$ & $25(12.4 \%)$ & $1(0.5 \%)$ & $1(0.5 \%)$ \\
\hline
\end{tabular}

Where, RMFR: Residues in most favoured regions, RAAR: Residues in additional allowed regions, RGAR: Residues in generously outlier regions, RDAR: Residues in disallowed regions.

\section{Validation}

The models were further validated by Ramachandran plot, by submitting the files to PDBsum. The model number 2 was endorsed as the residues in the most favoured region are $88.6 \%$ and the number of residues in additional allowed region is $10.9 \%$ (table 2).

\section{Loop modeling}

The PDB file format of model number 2 was recommended for loop optimization to Mod Loop (https://modbase.-compbio. ucsf.edu/modloop/) and the structure was validated with the help of Ramachandran plot obtained using PDBsum.

The model was validated as it had a maximum percentage (95 $\%$ ) of residues in most favoured region and $5 \%$ residues in additional allowed regions with no residues in generously allowed as well as disallowed regions (table 3).

The model of hK12 receptor protein (fig. 1) was successfully submitted to Protein model database (http://bioinformatics. cineca.it/PMDB/) bearing the PMDB ID: PM0080426.

Table 3: PROCHECK statistics

\begin{tabular}{llll}
\hline Ramachandran plot statistics* & & & G-factors** \\
\hline Regions & No. of Residues & Percentage & Parameters \\
\hline Most favoured regions [A, B, L] & 192 & 95.0 & Dihedral angles \\
Additional allowed regions [a, b, l, p] & 10 & 5.0 & Phi-psi distribution \\
Generously allowed regions $[\sim \mathrm{a}, \sim \mathrm{b}, \sim \mathrm{l}, \sim \mathrm{p}]$ & 0 & 0.0 & Chil-chi2 distribution \\
Disallowed regions [XX] & 0 & 0.0 & Chil only \\
Non-glycine and non-proline residues & 202 & 100 & Chi3 and chi4 \\
End residues (excl. Gly and Pro) & 2 & -0.24 & Omega \\
Glycine residues & 34 & - & Average Score \\
Proline residues & 16 & - & Main-chain covalent forces \\
Total no. of residues & 254 & - & Main-chain bond lengths \\
& & & Main-chain bond angles \\
& & & Average score \\
\end{tabular}

*Based on an analysis of 118 structures of resolution of at least 2.0 Angstroms and $R$-factor no greater than 20.0 a good quality model would be expected to have over $90 \%$ in the most favoured regions $[\mathrm{A}, \mathrm{B}, \mathrm{L}] .,{ }^{* *} \mathrm{G}$-factors provide a measure of how unusual, or out-of-the-ordinary, a property is., Values below-0.5-unusual, Values below-1.0-highly unusual. 


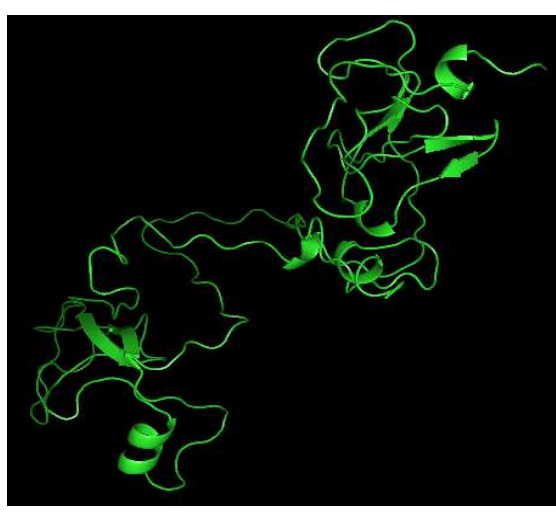

Fig. 1: Optimized model of hK12 receptor protein

\section{Ligand generation and docking}

A total of 236 drugs like compounds were downloaded from The Drug Bank based on their interaction with the FASTA sequence of the hK12 receptor protein. These were docked against hK12 protein using AutoDock Vina in PyRx 0.8. The results revealed that the lowest binding energy $(-11.6 \mathrm{Kcal} / \mathrm{mol})$ with hk12 protein is of ligand DB04786 (Suramin) having IUPAC name as 8-\{4-methyl-3[3-[\{[3-[\{2-methyl-5-[[4,6,8-trisulfonaphthalen-1-yl]carbamoyl] phenyl\}carbamoyl]phenyl]carbamoyl\}amino]benzamido]benzamido \}naphthalene-1,3,5-trisulfonic acid. The suramin falls under the category of antineoplastic agents, antinematodal agents and trypanocidal agents. The result suggested that the compound can be a promising ligand for the target hK12 protein.

\section{Ligand designing and docking}

The structural variation was done in the molecule DB04786 and 16 new compounds were designed with the help of ACD/ChemSketch 8.0 (fig. 2). The virtual screening of these compounds was done against hK12 receptor protein using AutoDock Vina in PyRx 0.8. The results indicated that out of all these compounds, ligand10, 8,8'-\{methanediylbis [imino[2,5-dimethylbenzene-3,1-diyl]methan-ediylimino[4-methylben zene-3,1-diyl]methanediylimino]\} dinaphthalene-1,3,5-trisulfonic acid, possesses the minimum binding energy ( $-12.3 \mathrm{Kcal} / \mathrm{mol}$ ) (fig. 3), which is greater than that of compound DB04786; other ligands like ligand16, 8,8'-\{methanediylbis[iminobenzene-3,1-diylmethan-ediylimino[4-aminobenzene-3,1-diyl]methanediylimino]\} dinaphthalene- 1,3,5-trisulfonic acid and ligand5, 8,8'-[methanediylbis[iminobenzene-3,1-diylmethanediylimino-benzene-3,1-iylmethanediylimino]] dinaphtha-lene-1,3,5trisulfonic acid also have the binding energy more favourable as compared to DB04786 and ligand6, $N, N^{\prime}$-bis $\{3-[[\{2-m e t h y l-5-$ [[naphthalen-1-lamino]methyl] phenyl\}amino]methyl] phenyl\} Methanediamine has binding energy comparable to ligand DB04786.

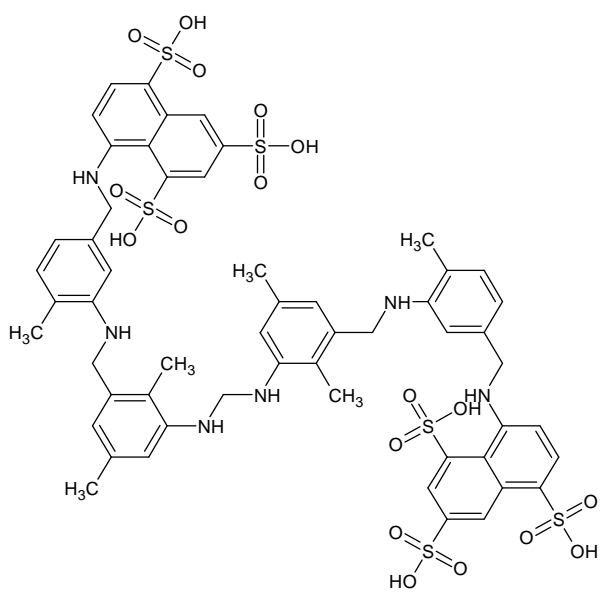

Ligand10

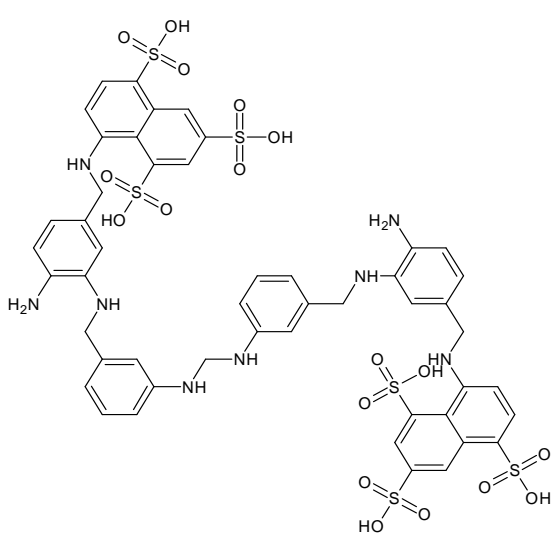

Ligand16

Fig. 2: The structures of suramin and other ligands

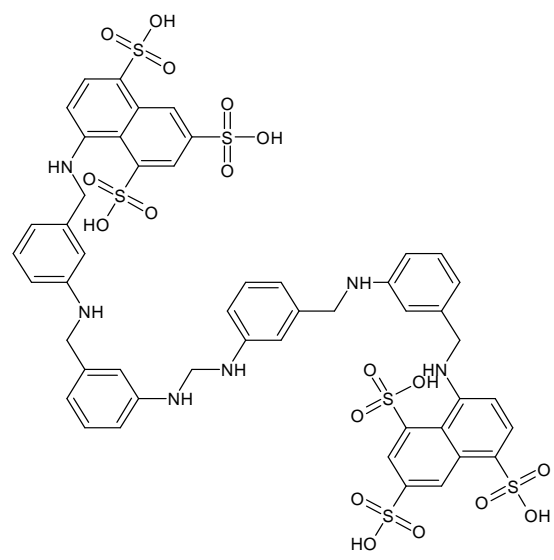

Ligand5

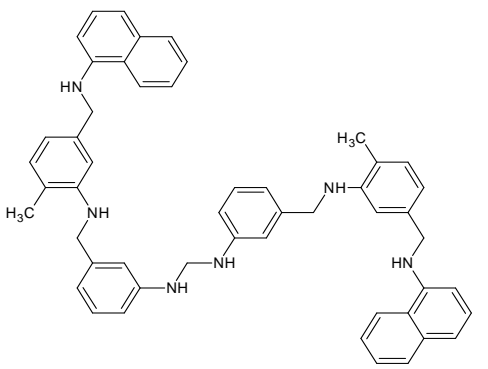

Ligand6

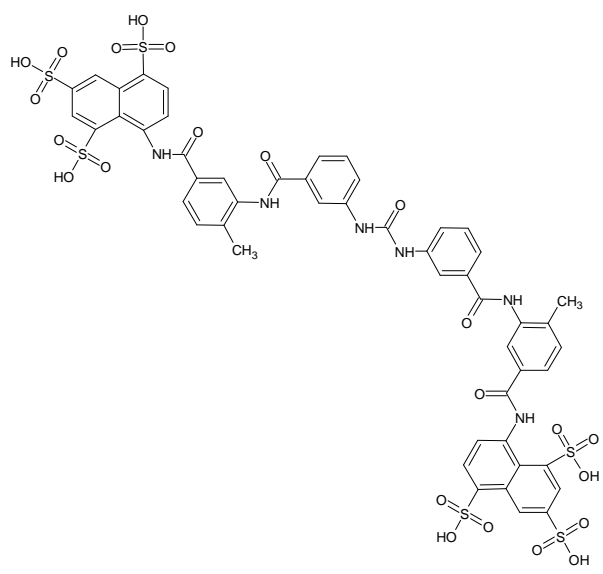

Suramin

Fig. 2: The structures of suramin and other ligands 


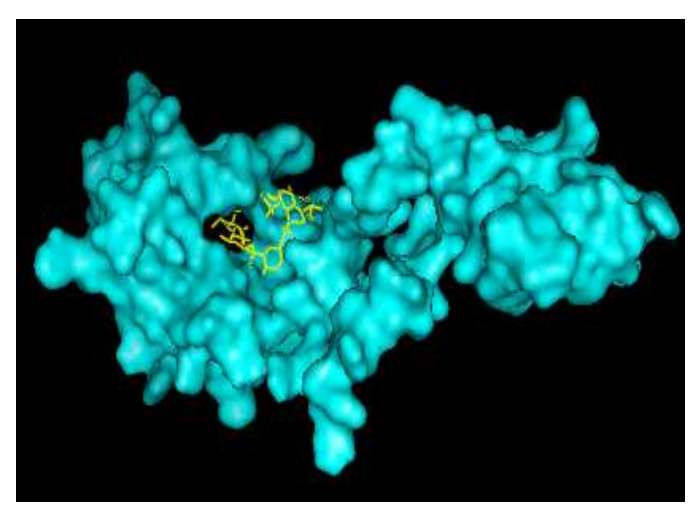

Fig. 3: Docking of ligand 10 with hK12 receptor protein

\section{CONCLUSION}

The techniques of homology modeling and loop modeling were utilized to design model of hK12 receptor protein. The model was validated by the Ramachandran plot. Various ligands were identified using Drug bank. The molecular docking done against hK12 receptor protein of these ligands using AutoDock Vina in PyRx 0.8 identified DB04786 with minimum binding energy $(-11.6 \mathrm{Kcal} / \mathrm{mol})$. The structure of this compound was varied by using ACD/ChemSketch 8.0 and then docking was done against the target protein. This study indicated that the ligand10 bears the minimum binding energy ($12.3 \mathrm{Kcal} / \mathrm{mol}$ ) with the target protein and thus the prospects of binding are high. The results of the present study clearly demonstrated that the in silico molecular docking studies of selected ligands, i.e., suramin, ligands 5, 6, 10 and 16 with hK12 protein exhibited favourable binding interactions and warranted further studies needed for the development of potent inhibitors for the overexpression of the hK12 protein. Further, investigations on the above compounds need in-vitro and in vivo studies to develop potential chemical entities for the prevention and treatment of gastric cancer.

\section{Funding}

This research did not receive any specific grant from funding agencies in the public, commercial, or not-for-profit sectors.

\section{CONFLICT OF INTERESTS}

Declare none

\section{REFERENCES}

1. Indian Council of Medical Research, Department of Health Research and Director General. Consensus document for management of gastric cancer. New Delhi: Division of Publication and Information, ICMR; 2014.

2. Waddell T, Verheij M, Allum W, Cunningham D, Cervantes A, Arnold D. Gastric cancer: ESMO-ESSO-ESTRO clinical practice guidelines for diagnosis, treatment and follow-up. Ann Oncol 2013;24:vi57-vi63.

3. Kanat O, Neil BO, Shahda S. Targeted therapy for advanced gastric cancer: a review of current status and future prospects. World J Gastrointest Oncol 2015;7:401-10.

4. Diamandis EP, Yousef GM. Human tissue kallikreins: a family of new cancer biomarkers. Clin Chem 2002;48:1198-205.

5. Borgoño CA, Diamandis EP. The emerging roles of human tissue kallikreins in cancer. Nat Rev Cancer 2004;4:876-90.

6. Kim H, Scorilas A, Katsaros D, Yousef GM, Massobrio M, Fracchioli S, et al. Human kallikrien gene 5 (KLK5) expression is an indicator of poor prognosis in ovarian cancer. Br J Cancer 2001;84:643-50.

7. Mavridis K, Scorilas A. Prognostic value and biological role of the kallilrein-related peptidases in human malignancies. Future Oncol 2010;6:269-85.

8. Wu Y, Lu M, Zhou Q. Kallikrein expression as a prognostic factor in ovarian cancer: a systematic review and metaanalysis. J Buon 2015;20:855-61.
9. Yang F, Li JY, Yin QN, Yang K, Dong SN, Bai LJ, et al. Human kallikrein 5 as a novel prognostic biomarker for triple-negative breast cancer: tissue expression analysis and relationship with disease course. Genet Mol Res 2015;14:9655-66.

10. Nagahara H, Mimori K, Utsunomiva T, Barnard GF, Ohira M, Hirakawa W, et al. Clinicopathologic and biological significance of kallikrein 6 overexpression in human gastric cancer. Clin Cancer Res 2005;11:6800-6.

11. Feng B, Xu WB, Zheng MH, Ma JJ, Cai Q, Zhang Y, et al. Clinical significance of human kallikrein 10 gene expression in colorectal cancer and gastric cancer. J Gastroenterol Hepatol 2006;21:1596-603.

12. Wen YG, Wang Q, Zhou CZ, Yan DW, Qiu GQ, Yang C, et al. Identification and validation of kallikrein-related peptidase 11 as a novel prognostic marker of gastric cancer based on immunohistochemistry. J Surg Oncol 2011;104:516-24.

13. Konstantoudakis G, Florou D, Mavridis K, Papadopoulos IN, Scorilas A. Kallikrein-related peptidase 13 (KLK13) gene expressional status contributes significantly in the prognosis of primary gastric carcinomas. Clin Biochem 2010;43:1205-11.

14. Memari N, Diamandis EP, Earle T, Campbell A, Van Dekken H, Van der Kwast TH. Human kallikrein-related peptidase 12: antibody generation and immunohistochemical localization in prostatic tissues. Prostrate 2007;67:1465-74.

15. Yousef GM, Magklara A, Diamandis EP. KLK 12 is a novel serine protease and a new member of the human kallikrein gene familydifferential expression in breast cancer. Genomics 2000;69:331-41.

16. Zhao EH, Shen ZY, Liu H, Jin X, Cao H. Clinical significance of human kallikrein 12 gene expression in gastric cancer. World J Gastroenterol 2012;18:6597-604.

17. Yang SSO, Lu JY, Kong XQ Liang ZJ, Luo C, Jiang H. Computational drug discovery. Acta Pharm Sinic 2012;33:1131-40.

18. Devgan M. Homology modelling and molecular docking studies of DNA replication licensing factor minichromosome maintenance protein 5 (MCM5). Asian J Pharm Technol 2015;5:17-22.

19. Devgan M. Structure prediction and in silico designing of drugs for the inhibition of Eph A10 tyrosine kinase receptor protein. Acta Biol Sci 2016;3:86-94.

20. Devgan M. Structure prediction and in silico designing of drugs against homeobox C8 protein. J Chem Pharm Res 2016;8:891-9.

21. Devgan M, Karar PK, Agarwal G, Mohan A, Gangwar P. In silico designing of drugs for the inhibition of AMF-HER2 complex in trastuzumab-resistant breast cancer. Indian J Biotechnol 2016;15:292-8.

22. Wikipedia. The free encyclopedia [Internet]. San Franscisco (CA): Wikipedia Foundation, Inc (US); c2001-16. Available from: https://en.wikipedia.org/wiki/FASTA_format. [Last accessed on 19 Jan 2016]

23. National Center for Biotechnology Information [Internet]. Bethesda (MD): National Library of Medicine (US); Kallikrein 12 [Homo sapiens]. Available from: http:// www.ncbi.nlm.nih.gov/rotein/10799397?report=fasta. [Last accessed on 22 Oct 2015]

24. Wikipedia. The free encyclopaedia [Internet]. San Franscisco (CA): Wikipedia Foundation, Inc (US); 2001-2016. BLAST. Available from: https://en.wikipedia.org/wiki/BLAST. [Last accessed on 19 Jan 2016].

25. National Center for Biotechnology Information [Internet]. Bethesda (MD): National Library of Medicine (US); BLASTP. Available from: http://blast.ncbi.nlm.nih.gov/Blast.cgi?PROGRAM=blastandPAGE_ TYPE=BlastSearch. [Last accessed on 23 Oct 2015]

26. Kelly L. 3D-PSSM. South Kensington Campus (London): Imperial College of Science, Technology and Medicine (UK); c1999. Available from: http://sbg.bio.ic.ac.uk/ 3dpssm/index2.html. [Last accessed on 23 Oct 2015]

27. Kelly LA, Sternberg MJ. Protein structure prediction on the web: a case study using the Phyre server. Nat Protoc 2009;4:363-71.

28. Kelly L, Sternberg M. Phyre2 [Internet]. South Kensington Campus (London): Structural Bioinformatics Group, Imperial College (UK). Available from: http://www.sbg.bio.ic.ac.uk/ $\sim$ phyre $2 / \mathrm{html} /$ page.cgi?id=index. [Last accessed on 23 Oct 2015].

29. Research Collaboratory for Structural Bioinformatics. RCSB Protein Data Bank [Internet]. New Jersey, California: Rutgers, UCSanDiego 
(US). Available from: http://www.rcsb.org/pdb/home.do. [Last accessed on 24 Oct 2015]

30. Kuntal BK, Aparoy P, Reddanna P. EasyModeller: a graphical interface to modeller. BMC Res Notes 2010;3:226.

31. Kuntal BK. EasyModeller (Version 2.0) [Internet]. Hyderabad: Kuntal Kumar Bhusan, University of Hyderabad (India). Available from: http://www.sites.google.com/site/bioinformatikz. [Last accessed on 08 Apr 2015]

32. Duex N, Peitsch M, Schwede T, Diemand A. DeepView/SwissPdbViewer [Internet]. Bazel: Structural Bioinformatics Groups, Swiss Institute of Bioinformatics, Biozentrum University of Bazel (Switzerland); c1995-2001. Available from: http://Spdbv.vital-it.ch/ [Last accessed on 06 Apr 2015]

33. European Bioinformatics Institute (EMBL-EBI). PDBsum [Internet]. Hinxton, Cambridgeshire: Wellcome Trust Genome Campus (UK); cEMBL-EBI; 2016. Available from: https://www.ebi.ac.uk/ thornton-srv/databases/cgi-

bin/pdbsum/GetPage.pl?pdbcode=index.html. [Lase accessed on 24 Oct 2015]

34. Laskowski RA. PDBsum new things. Nucleic Acids Res 2009;37:D355-9.

35. Fiser A, Do RK, Sali A. Modeling of loops in protein structures. Protein Sci 2000;9:1753-73.

36. Fiser A, Sali A. ModLoop: automated modelling of loops in protein structures. Bioinformatics 2003;19:2500-1.

37. Fiser A. ModLoop [Internet]. San Francisco (CA): University of California (USA); c2000. Available from: http://modbase. compbio.ucsfedu/modloop/. [Last accessed on 25 Oct 2015]

38. Bakker PD, Lovell S. RAMPAGE [Internet]. Cambridge (UK): Department of Biochemistry, University of Cambridge. Available from: http://mordred.bioc.cam.ac.uk/ rapper/rampage.php. [Last accessed on 25 Oct 2015]

39. Wishart DS, Knox C, Guo AC, Shrivastava S, Hassanali M, Stothard P, et al. DrugBank: a comprehensive resource for in silico drug discovery and exploration. Nucleic Acids Res 2006;34:D668-72.

40. Wishart DS. DrugBank [Internet]. Canada: Canadian Institutes of Health Research, Alberta Innovates-Health Solutions, The Metabolomics Innovation Centre. Available from: http:// www.drugbank.ca. [Last accessed on 28 Oct 2015]

41. Vyas V, Jain A, Jain A, Gupta A. Virtual screening: a fast tool for drug design. Sci Pharm 2008;76:333-60.

42. Forli S. Charting a path to success in virtual screening. Molecules 2015;20:18732-58.

43. PerkinElmer. ChemBio3D. Waltham, Massachusetts: PerkinElmer Inc. (USA); c1998-2016. Available from: http:// www.cambridgesoft.com/Ensemble for chemistry/ chemBio3D/. [Last accessed on 02 Nov 2015]

44. Schrödinger. PyMol. New York: Schrödinger (USA); c2005-16. Available from: http://www.pymol.org. [Last accessed on 02 Nov 2015].

45. Dallakyan S, Olson AJ. Small-molecule library screening by docking with PyRx. Methods Mol Biol 2015;1263:243-50.

46. Dallakyan S. Python prescription, a virtual screening tool. New York: Source Forge, Slashdot Media, DHI Group Inc. (USA); C2009-16. Available from: http://pyrx.sourceforge.net/. [Last accessed on 11 Dec 2015].

\section{How to cite this article}

- Manish Devgan. Structure prediction and in silico designing of drugs against kallikrein protein 12. Int J Curr Pharm Res 2017;9(2):64-69. 